Annuaire suisse de politique de développement

22-2 | 2003

Société de l'information et coopération internationale

\title{
E-commerce : du mirage économique au miracle social
}

Benoît Vulliet

\section{(2) OpenEdition}

1 Journals

Édition électronique

URL : http://journals.openedition.org/aspd/540

DOI : $10.4000 /$ aspd. 540

ISSN : 1663-9669

Éditeur

Institut de hautes études internationales et du développement

Édition imprimée

Date de publication : 1 novembre 2003

Pagination : 39-41

ISSN : 1660-5934

\section{Référence électronique}

Benoît Vulliet, «E-commerce : du mirage économique au miracle social », Annuaire suisse de politique de développement [En ligne], 22-2 | 2003, mis en ligne le 22 mars 2010, consulté le 08 septembre 2020. URL : http://journals.openedition.org/aspd/540; DOI : https://doi.org/10.4000/aspd.540

(c) The Graduate Institute I Geneva 


\title{
E-commerce: du mirage économique au miracle social
}

\author{
Benoît Vulliet*
}

\section{Commerce électronique}

Dans les pays où Internet est largement accessible, une vision s'est développée: avec près de 5 millions d'entreprises micro, petites et moyennes, le commerce électronique semble s'imposer comme la solution de tous les problèmes. L'utilisateur consommateur se voit proposer un éventail sans fin de biens et de services, au point de croire que sa vie dépend d'Internet. Pour l'utilisateur entrepreneur, la voie électronique devient incontournable, que ce soit pour proposer des produits ou faire des transactions.

En fait, $8 \%$ de la population mondiale a accès à Internet, dont $50 \%$ dans les pays les plus riches ${ }^{1}$. Le commerce électronique ne représente que $6 \%$ des parts du marché mondial. Les pays en développement se partagent moins de $5 \%$ de ces $6 \%$, qui sont répartis ainsi: Asie et Pacifique 83,5\%, économies en transition $8,7 \%$, Amérique latine $7,3 \%$, et Afrique, le parent pauvre, $0,5 \%$ (ou environ 500 millions de dollars) $)^{2}$.

D'autre part, si le commerce électronique est très diversifié ${ }^{3}$, l'essentiel des échanges - 95\% - se fait entre entreprises (B2B), ne laissant donc que $5 \%$ aux entreprises proposant au consommateur l'achat de biens ou services directement par Internet (B2C) ${ }^{4}$. Parmi ces dernières, les plus rentables sont celles qui vendent en ligne des livres, des vidéos et des DVD. Dans les pays en développement, elles vendent surtout des services touristiques ou de l'artisanat, mais à des consommateurs se trouvant dans les pays développés, où elles installent aussi leurs serveurs informatiques pour bénéficier de bonnes conditions d'exploitation, comme la stabilité et la rapidité des lignes ainsi que des services de paiement sécurisés.

* Chargé d'enseignement à l'Institut universitaire d'études du développement (iuéd), Suisse.

1 Barayre-El Shami Cécile, Building Up New Business Models for Digital Inclusion, ITU/IDSC Regional Seminar on E-business for the Arab Region, Cairo, Egypt, 10-12 December 2002, <www.ebi.org. $\mathrm{eg} / \mathrm{E}>$.

2 Selon la CNUCED, E-commerce and Development Report 2002, pp. 6-8. Ce rapport indique que les parts du marché du commerce électronique devraient passer de $6 \%$ en 2002 à $18 \%$ en 2006.

3 Cela donne lieu à l'utilisation de nombreux mots-valises comme B2B (business to business, ou une transaction commerciale se faisant entre deux entreprises, p. ex. la sous-traitance...), B2C (business to consumer, ou une entreprise qui vend en ligne des biens et des services au consommateur final), B2G (business to government, ou une entreprise travaillant pour une administration publique), C2C (commerce entre consommateurs), etc.

4 CNUCED, op. cit., p. 8. 
Ainsi, le commerce électronique est surtout le fait de relations entre entreprises dans les pays riches; ailleurs, il est très marginal. Toutefois, depuis quelques années, des entreprises d'un genre nouveau ont émergé, exploitant une niche particulière, le «marché de la diaspora»: $\mathrm{B} 2 \mathrm{D}$ ou business to diaspora.

\section{Le marché de la diaspora}

Le marché de la diaspora est exploité par deux types d'entreprises: d'une part, celles qui fournissent des biens et des services aux expatriés se trouvant dans les pays développés, ou business-to-diaspora consumer marketing $^{5}$, comme les voyages, le logement, l'envoi d'argent, de produits alimentaires, symboliques, religieux, etc., provenant des pays d'origine; d'autre part, celles qui vendent et distribuent dans un pays en développement des produits achetés en ligne par les expatriés, ou des personnes ayant un lien avec ce pays, pour leurs famille et amis. Dans ce cas, il y a trois acteurs, d'où le terme de split marketing $^{6}$ : le vendeur dans un pays en développement, l'acheteur dans un pays développé, le bénéficiaire dans le même pays en développement. A la tête de ces entreprises (le vendeur), on trouve le plus souvent des personnes qui se sont expatriées pendant un certain temps dans des pays développés pour y travailler ou étudier, et qui reviennent dans leur pays pour mettre à profit leurs compétences, notamment des femmes qui peuvent ainsi créer des entreprises tout en assumant leurs activités familiales ${ }^{7}$.

On peut ainsi lire, à propos d'une entreprise péruvienne vendant des gâteaux de fête ${ }^{8}$ : «Il est possible de démarrer une entreprise de commerce électronique couvrant tout un pays, même avec un produit aussi simple que des gâteaux faits maison. De simples ménagères peuvent apprendre très rapidement à se servir d'Internet pour recevoir des commandes. Ces femmes peuvent combiner la préparation des gâteaux et l'emploi d'Internet avec le soin qu'elles doivent donner à leurs enfants sans devoir travailler hors de chez elles. L'avènement de l'information électronique et des ordinateurs est en train de changer le monde et la manière de commercer. Les habitants des pays en développement doivent saisir l'occasion et utiliser leur imagination et leur créativité pour générer travail et revenus. Notre modèle de commerce électronique est simple et bon marché à mettre en place; il peut être reproduit dans d'autres pays et avec d'autres produits. Il suffit d'un peu d'imagination, de travail et d'ouverture à la technologie pour y arriver. ${ }^{9}$

Si le principe est simple, sa mise en œuvre l'est-elle aussi? Ces entreprises sont-elles alors économiquement rentables? Bien souvent, ces femmes et hommes entrepreneurs ne se sont pas doutés de la difficulté de

5 Selon l'Economic Commission for Africa, The African Development Forum '99: Post ADF Summit. Electronic Commerce in Africa, 1999, <www.uneca.org/adf99/adf99ecommerce.htm>.

Ibid.

CNUCED, op. cit., p. 64.

$<w w w . t o r t a s p e r u . c o m . p e>$

« Ordinateurs et gâteaux donnent confiance et argent aux ménagères péruviennes », Forum du commerce international. La revue du Centre de commerce international, $\mathrm{n}^{\mathrm{o}} 3,2000$,

$<$ www.forumducommerce.org/news/fullstory.php/aid/169>, p. 30. 
la tâche et des contraintes imposées par ce type de commerce.

\section{Difficultés et solutions}

En effet, ces contraintes sont celles que rencontre tout commerce électronique en relation avec les pays en développement, comme

$\square \quad$ les aspects légaux et la réglementation: cryptage-décryptage, signature digitale, organismes de certification, protection des consommateurs, déficience des tribunaux, droits de propriété intellectuelle, réglementations et procédures commerciales ;

$\square$ les aspects financiers: paiements électroniques, banques locales n'acceptant pas les transactions ;

$\square$ les aspects technologiques: infrastructure des télécommunications (réseau et bande passante), équipement des personnes (ménages et entreprises), coûts élevés des communications ;

$\square$ les aspects logistiques: procédures de dédouanement, stockage et distribution physique ${ }^{10}$.

Concernant précisément le split marketing, les deux contraintes majeures sont la capacité de distribuer des produits diversifiés dans tout le pays et la capacité de gérer les paiements par carte de crédit, ce qui limite leur rentabilité (entre 2000 et 30'000 dollars par an), malgré les sommes importantes transférées par les diasporas (p. ex., pour l'Ethiopie, 100 millions de dollars par an $)^{11}$.

L'accumulation des problèmes et la faible rentabilité n'empêchent pourtant pas ces entreprises à la fois de continuer à évoluer et d'augmenter en nombre. Les responsables cherchent de nouvelles sources de financements, s'adressant aux ministères, agences internationales et nationales de coopération, ONG, mettant en avant l'utilité sociale de leur entreprise: création d'emplois, formation, valorisation des compétences, renforcement des liens familiaux et interpersonnels. Ainsi, le caractère économiquement rentable de ces entreprises ne repose pas sur les échanges financiers très importants liés à la diaspora, mais sur leur capacité à valoriser leur dimension socialement utile.

Si le commerce électronique en général est effectivement insignifiant en regard du volume mondial des transactions, celui qui met à profit le marché de la diaspora représente localement un potentiel de développement important. Ce potentiel est largement fondé sur l'ingéniosité et l'inventivité de femmes et d'hommes qui se lancent dans une aventure dont l'impact social est significatif. Toutefois, la nature de cet impact n'est pas encore connue, pas plus que celle de ses causes réelles, et cela constitue encore une question pour la recherche.

10 Selon l'Economic Commission for Africa, op. cit.

11 Barayre-El Shami Cécile, Information and Communication Technologies: Opportunities and Challenges for SMEs in Developing Countries, Latin American and Caribean Symposium on Education, Science and Culture in the Information Society, Cuba, 18-21 February 2002, 13 p., <http://espejos. unesco.org.uy/simplac2002/Ponencias/SIMPLAC/SL057.doc>. 